\title{
Convergence and Interaction of Hippocampal and Amygdalar Projections within the Prefrontal Cortex in the Rat
}

\author{
Akinori Ishikawa and Shoji Nakamura \\ Department of Neuroscience, Yamaguchi University School of Medicine, Ube, Yamaguchi, 755-8505, Japan
}

\begin{abstract}
The orbital and medial prefrontal cortex (OMPFC) receives inputs from the CA1/subicular (CA1/S) region of the ventral hippocampus and the basolateral nucleus of the amygdala (BLA). Despite many studies about these projections, little is known as to how CA1/S and BLA inputs converge and interact within the OMPFC. Extracellular recordings of single-unit activity in the OMPFC were performed in sodium pentobarbitone-anesthetized rats. OMPFC neurons driven by CA1/S or BLA stimulation were more frequently encountered in the ventral portion of the prelimbic (v-PrL) and infralimbic cortex (IL). OMPFC neurons showing excitatory convergence of both inputs from the CA1/S and BLA were also located predominantly in the v-PrL and IL. The excitatory latencies of these neurons from both the CA1/S and BLA revealed almost identical values. Excitatory responses of OMPFC neurons to CA1/S (or BLA) stimulation were markedly augmented by simultaneous BLA (or CA1/S) stimulation, whereas the inhibitory influence of the BLA (or CA1/S) on CA1/S-induced (or BLA-induced) excitation was apparent when BLA (or CA1/S) stimulation was given 20 - 40 msec before CA1/S (or BLA) stimulation. Similar results were also observed when reciprocal connections between the CA1/S and BLA were severed to exclude the influences of these connections on one another. From these studies, we concluded that excitatory and inhibitory inputs from the hippocampus and amygdala converge and interact in the v-PrL and IL. Furthermore, the results indicate that simultaneous activation of hippocampal and amygdalar neurons may be important for amplification of OMPFC neuronal activity.
\end{abstract}

Key words: prefrontal cortex; infralimbic cortex; hippocampus; amygdala; memory; emotion

\section{Introduction}

The medial portion of the prefrontal cortex, including the orbital and medial prefrontal cortex (OMPFC), is implicated in both learning and memory processes (Kesner et al., 1996; Ragozzino et al., 1998; Baldwin et al., 2002) and in regulation of emotional behaviors (Morgan et al., 1993; Jinks and McGregor, 1997; Quirk et al., 2000). Previous studies have demonstrated that the OMPFC plays a crucial role as an interface between the limbic and neocortical regions of these processes, given that the OMPFC receives inputs from various limbic structures and is reciprocally related to the sensory association cortices in the parietal and temporal lobes (Sesack et al., 1989; Takagishi and Chiba, 1991; Condé et al., 1995). Two major limbic structures, the CA1/subicular (CA1/S) region of the ventral (V) hippocampal formation (Jay and Witter, 1991; Thierry et al., 2000) and the basolateral nucleus of the amygdala (BLA) (Krettek and Price, 1977b; McDonald, 1987; Bacon et al., 1996), project directly to the OMPFC. The $\mathrm{CA} 1 / \mathrm{S}$ projection to the OMPFC is implicated in memory processes such as delayed radial arm maze tasks (Floresco et al., 1997), whereas the amygdalar OMPFC circuit is thought to be

Received June 4, 2003; revised Sept. 10, 2003; accepted Sept. 10, 2003.

This work was supported by Japan Health Sciences Foundation Grant SH24207. We are grateful to Prof. N. Harada for helpful advice about data analysis, and K. Tanaka and M. Ogura for their secretarial work.

Correspondence should be addressed to Shoji Nakamura, Department of Neuroscience, Yamaguchi University School of Medicine, 1-1-1 Minamikogushi, Ube, Yamaguchi, 755-8505, Japan. E-mail: snaka@yamaguchi-u.ac.jp. Copyright $\odot 2003$ Society for Neuroscience $\quad 0270-6474 / 03 / 239987-09 \$ 15.00 / 0$ involved in classical fear conditioning (Garcia et al., 1999) and reward memory (Gaffan et al., 1993).

Previous anatomical studies (Krettek and Price, 1977b; Jay and Witter, 1991; Condé et al., 1995) have demonstrated that the CA1/S and BLA projections to the OMPFC innervate predominantly the prelimbic (PrL) and infralimbic cortex (IL). Electrophysiological studies (Laroche et al., 1990; Jay et al., 1992) also revealed that the PrL and IL are the major targets of the CA1/S projections. However, little is known about how inputs to the OMPFC from the hippocampus and amygdala converge and interact within the OMPFC. Considering that learning acquisition and memory consolidation are reinforced by the presence of a reward, the OMPFC may be an important brain site for regulation of memory consolidation processing in relationship to emotion. In view of the modulatory effects of emotional information from the amygdala on hippocampal signal processing within the OMPFC, it is important to investigate the convergence and interaction of hippocampal and amygdalar inputs within the OMPFC.

In the present experiment, we examined the electrophysiological properties and topographical organization of OMPFC neurons responding to stimulation of the hippocampus and amygdala. The pattern of convergence and segregation of the two inputs within the OMPFC was also studied. In addition, after lesioning the reciprocal connections between the hippocampus and amygdala, the effects of conditioning stimulation of the amygdala (or hippocampus) on hippocampal (or amygdalar) ex- 
Table 1. Responses of OMPFC neurons to CA1/S and BLA stimulation

\begin{tabular}{|c|c|c|c|c|c|}
\hline \multirow[b]{2}{*}{ Stimulated sites } & \multicolumn{5}{|c|}{ Recording sites } \\
\hline & $\begin{array}{l}\mathrm{Cg} 1 \\
(n=22)\end{array}$ & $\begin{array}{l}d-\operatorname{PrL} \\
(n=29)\end{array}$ & $\begin{array}{l}\mathrm{v}-\operatorname{PrL} / \mathrm{M} 0 \\
(n=48)\end{array}$ & $\begin{array}{l}\mathrm{IL} \\
(n=31)\end{array}$ & $\begin{array}{l}\text { DP/V0 } \\
(n=10)\end{array}$ \\
\hline \multicolumn{6}{|l|}{ Excitation } \\
\hline CA1/S & $3 / 22(14 \%)$ & $4 / 29$ (14\%) & $20 / 48(42 \%)$ & $19 / 31(61 \%)$ & $5 / 10(50 \%)$ \\
\hline BLA & $3 / 22(14 \%)$ & $3 / 29(10 \%)$ & $6 / 48(13 \%)$ & $11 / 31(35 \%)$ & $3 / 10(30 \%)$ \\
\hline $\mathrm{CA} 1 / \mathrm{S}+\mathrm{BLA}$ & $1 / 22(5 \%)$ & $1 / 29(3 \%)$ & $5 / 48(10 \%)$ & $10 / 31(32 \%)$ & $1 / 10(10 \%)$ \\
\hline \multicolumn{6}{|l|}{ Inhibition } \\
\hline $\mathrm{CA} 1 / \mathrm{S}$ & $7 / 11(64 \%)$ & $5 / 9(56 \%)$ & $8 / 11(73 \%)$ & $7 / 7(100 \%)$ & $1 / 2(50 \%)$ \\
\hline BLA & $8 / 10(80 \%)$ & $5 / 5(100 \%)$ & $6 / 7(86 \%)$ & $5 / 7(71 \%)$ & $0 / 2(0 \%)$ \\
\hline $\mathrm{CA} 1 / \mathrm{S}+\mathrm{BLA}$ & $5 / 9(56 \%)$ & $3 / 5(60 \%)$ & $5 / 7(71 \%)$ & $5 / 6(83 \%)$ & $0 / 1(0 \%)$ \\
\hline
\end{tabular}

The numbers of neurons recorded in each subregion of the OMPFC are presented in the parentheses under each recording site. The denominators show the numbers of neurons tested for excitatory or inhibitory response, and the numerators indicate those that revealed excitatory or inhibitory response.

citatory inputs were investigated. A portion of these data has been presented previously (Ishikawa and Nakamura, 2002).

\section{Materials and Methods}

Surgery and placement of electrodes. The experiments were reviewed and approved by the Yamaguchi University School of Medicine Committee of Ethics on Animal Experiments. Sprague Dawley rats were housed with free access to food and water at a constant $22^{\circ} \mathrm{C}$ and under a $12 \mathrm{hr}$ light/dark cycle (lights were on at 8:00 A.M., and off at 8:00 P.M.). Thirtyfive male rats $(270-560 \mathrm{gm})$ were anesthetized with sodium pentobarbitone $(50 \mathrm{mg} / \mathrm{kg}$, i.p.). Rats were supplemented with additional injections as necessary during experiments. Supplemental doses were $0.1-0.2 \mathrm{ml}$ of sodium pentobarbitone $(50 \mathrm{mg} / \mathrm{ml})$, which were injected intraperitoneally. The doses and timing of supplemental injections were determined by the EEG of recording sites and responses to the tail pinch. Body temperature was monitored by a rectal probe and maintained at $36-37^{\circ} \mathrm{C}$ using an electrical type of heating pad placed under the rat. The head was fixed horizontally in a stereotaxic instrument (Narishige, Tokyo, Japan). The skull was exposed, and a small burr hole was made at the appropriate coordinates to enable vertical penetration by the stimulating and recording electrodes. All electrodes were zeroed at bregma, and all coordinates were calculated from this point. The depth was calibrated from the cortical surface. Stereotaxic coordinates were determined according to the rat brain atlas of Paxinos and Watson (1998). Bipolar electrodes consisting of paired insulated stainless steel wires (diameter, $0.2 \mathrm{~mm}$ ) with an exposed tip of $\sim 0.1 \mathrm{~mm}$ were implanted for electrical stimulation of the $\mathrm{CA} 1 / \mathrm{S}$ region of the ventral hippocampus [posterior $(\mathrm{P}), 6.5 \mathrm{~mm}$; lateral (L), $5.4 \mathrm{~mm} ; \mathrm{V}, 3.0-6.0 \mathrm{~mm}$ ] and the BLA (P, 2.8-3.3 mm; L, $5.0 \mathrm{~mm} ; \mathrm{V}$, $7.3-8.0 \mathrm{~mm})$.

Lesions of reciprocal connections between the CA1/S and BLA. A stainless-steel needle (diameter, $0.2 \mathrm{~mm}$ ) was inserted vertically to the base of the brain at the following coordinates: P, 4.8 -5.3 mm; L, 6.0-7.0 $\mathrm{mm}$. The needle was swept in an arc mediolaterally, taking the point of the needle at the cortical surface as the center. This manipulation was repeated until field potentials in the CA1/S (or BLA) evoked by BLA (or $\mathrm{CA} 1 / \mathrm{S}$ ) stimulation $<0.3 \mathrm{~mA}$ (duration, $0.1 \mathrm{msec}$ ) disappeared.

Electrophysiological recording. Single-unit activity in the OMPFC [anterior (A), 2.7-4.0 $\mathrm{mm}$; $\mathrm{L}, 0.4-1.0 \mathrm{~mm} ; \mathrm{V}, 1.5-6.0 \mathrm{~mm}$ ] was recorded using a glass micropipette (5-10 $\mathrm{M} \Omega$ impedance) filled with $0.5 \mathrm{M}$ sodium acetate containing 2\% Pontamine Sky Blue. Field potentials in the CA1/S and BLA were recorded through a single stainless-steel wire of a bipolar electrode, which was the same as that used for stimulation of the CA1/S and BLA. For recording field potentials in the OMPFC (A, 2.7-3.2 $\mathrm{mm} ; \mathrm{L}, 0.75 \mathrm{~mm} ; \mathrm{V}, 3.5-4.0 \mathrm{~mm}$ ) evoked by CA1/S and BLA stimulation, the same type of electrode for stimulation of the CAl/S and BLA was used. The spikes and field potentials were bandpass-filtered (150-3000 $\mathrm{Hz}$ and $0.08-10000 \mathrm{~Hz}$, respectively), amplified (MEG-2100; Nihon Kohden, Tokyo, Japan), and digitized by way of a CED 1401 interface (Cambridge Electronics Design, Cambridge, UK). To investigate the firing probability, latency, and threshold of recorded neurons, electrical stimuli were delivered as a single pulse with a duration of $0.1 \mathrm{msec}$ at $0.5 \mathrm{~Hz}$ (orthodromic, 0.8-10.0 V or 0.03-1.00 mA; antidromic, 0.03-5.00 mA). In experiments in which the thresholds for antidromic and orthodromic responses to BLA stimulation were compared, threshold currents required for antidromic or orthodromic activation were determined. The following criteria were used to discriminate spikes antidromically driven by BLA stimulation: a fixed latency at the threshold for activation, the ability to follow twin pulse stimulation at short intervals (shorter than 3 $\mathrm{msec}$ ), and collision with spontaneous spikes for spontaneously active cells. Neurons were considered to be inhibited when a complete suppression of spontaneous activity was observed for $>25 \mathrm{msec}$. In a subset of OMPFC neurons $(n=44)$, to examine the effects of BLA (or CA1/S) stimulation on excitatory responses to CA1/S (or BLA) stimulation, test stimuli to the CA1/S (or BLA) were applied at various intervals (excitatory, $0,5,10$, and $20 \mathrm{msec}$; inhibitory, $0,20,40,80,120$, and $160 \mathrm{msec}$ ) after conditioning stimuli to the BLA (or CA1/S) (BLA-CA1/S and CA1/ S-BLA conditioning, respectively). To test the neurons responding to both CA1/S and BLA stimulation, subthreshold stimuli to the BLA or CA1/S (0.06-0.30 mA) were used. In neurons driven only by CA1/S (or BLA) stimulation, the conditioning stimuli to BLA (or CA1/S) were delivered at currents ranging from 0.16 to $0.30 \mathrm{~mA}$ with a $0.1 \mathrm{msec}$ duration.

Histology. Recording sites of single-unit activity were marked by microiontophoretic application of Pontamine Sky Blue dye (10 $\mu \mathrm{A} ; 5 \mathrm{~min})$, which was delivered through the tip of the recording electrodes. To mark sites of recording and stimulating through bipolar electrodes, a direct current of $30 \mu \mathrm{A}$ was delivered to the CA1/S, BLA, and OMPFC for 1 $\mathrm{min}$. At the end of each experiment, animals were deeply anesthetized by an additional injection of sodium pentobarbitone $(50 \mathrm{mg} / \mathrm{kg}$, i.p.), perfused with $4 \%$ paraformaldehyde solution, and the brains were removed for histology. Frozen coronal serial sections $(50 \mu \mathrm{m})$, including the OMPFC, ventral hippocampus, or BLA, were stained with HematoxylinEosin. Recording and stimulating sites were reconstructed according to the rat brain atlas of Paxinos and Watson (1998). In 35 animals, only those $(n=29)$ with correct positioning of the electrodes or successful lesion of the connection between the CA1/S and BLA were included in later analyses.

Data analysis. All data are expressed as mean \pm SEM. The data from two groups were analyzed by unpaired Student's $t$ test, and those from more than two groups were analyzed by repeated-measures (see Fig. $5 B, C$ ) or one-way ANOVA (see Fig. $6 B$ ) followed by Scheffé's post hoc test. Differences are considered significant at $p<0.05$.

\section{Results}

A total of 140 single units (15 animals) were recorded in the OMPFC. Recorded OMPFC neurons were located in area 1 of the cingulate cortex $(\mathrm{Cg} 1 ; n=22), \operatorname{PrL}(n=73)$, IL $(n=31)$, dorsal peduncular cortex (DP; $n=4)$, medial orbital cortex (MO; $n=$ $4)$, and ventral orbital cortex (VO; $n=6$ ) (Table 1$)$. The PrL was divided into two half subregions, dorsal (d-PrL; $n=29)$ and ventral $(\mathrm{v}$-PrL, $n=44)$ portions of the $\operatorname{PrL}$, on the basis of a previous study which reported that the d-PrL and $v-\operatorname{PrL}$ had different innervation patterns of hippocampal projection (Jay and Witter, 1991). The firing rates $(0.3 \pm 0.1$ spikes $/ \mathrm{sec} ; n=42)$ of neurons driven by CA1/S or BLA stimulation were lower than 


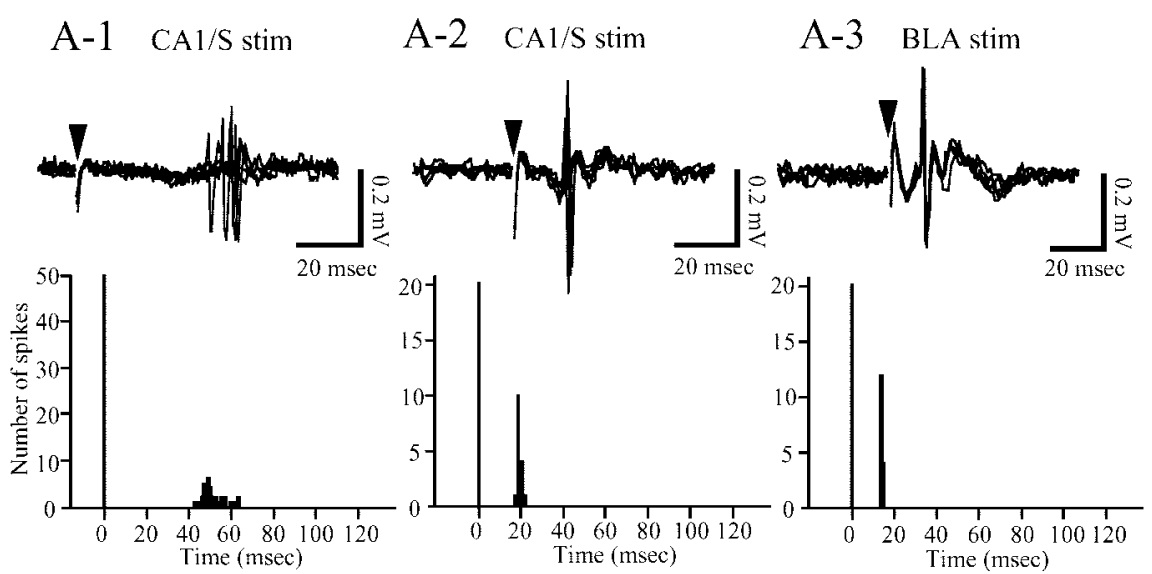

B-1 CAl/S stim

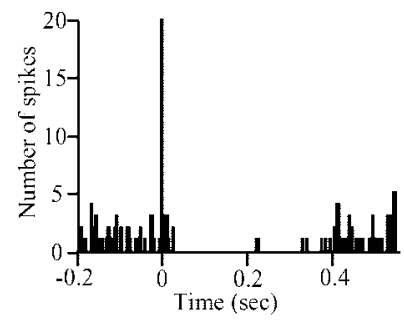

B-2 BLA stim

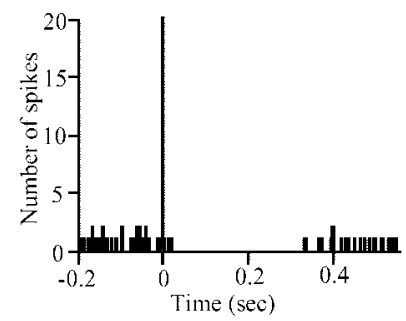

C BLA stim
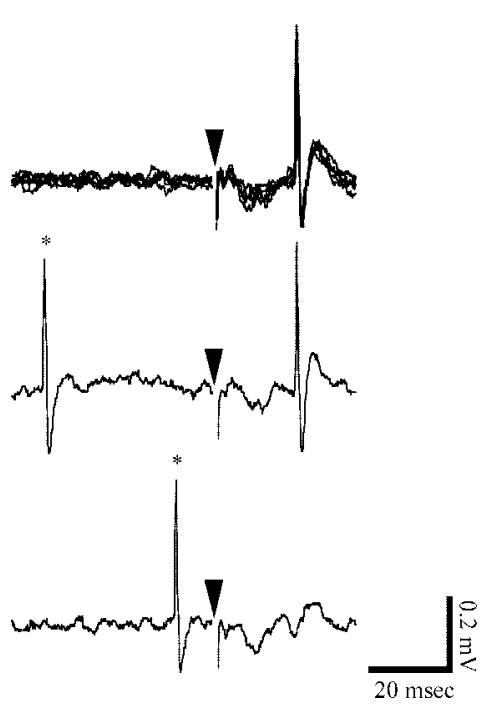

Figure 1. $A-C$, Excitatory $(A)$ and inhibitory $(B)$ responses of OMPFC neurons to CA1/S or BLA stimulation, and antidromic responses ( $C$ ) of OMPFC neuron to BLA stimulation. $A$, Top, Evoked spikes resulting from $C A 1 / S(A-1, A-2)$ and $B L A(A-3)$ stimulation. An OMPFC neuron $(A-1)$ located in the d-PrL showed more variable latencies compared with those of a neuron $(A-2)$ in the $\mathrm{v}$-PrL. The excitatory latencies of these neurons were $50 \mathrm{msec}(A-1)$ and $18 \mathrm{msec}(A-2)$, respectively. BLA stimulation excited a $\mathrm{v}$-PrL neuron $(A-3)$ at a latency of $14 \mathrm{msec}$. Arrowheads indicate stimulus artifacts. In all traces, five sweeps are superimposed. $A$, Bottom, Peristimulus time histograms (PSTHs) of the evoked responses presented in the top. Each PSTH was compiled by $50(A-1)$ and $20(A-2, A-3)$ sweeps with a $1 \mathrm{msec}$ bin width. $B$, Inhibitory responses of $\mathrm{v}$-PrL $(B-1)$ and IL $(B-2)$ neurons to CA1/S and BLA stimulation. Each PSTH was compiled of $39(B-1)$ and $55(B-2)$ sweeps with a 5 msec bin width. Both neurons revealed a prolonged, complete inhibition lasting $195 \mathrm{msec}(B-1)$ and $310 \mathrm{msec}(B-2)$, respectively. In all PSTHs in $A$ and $B$, stimuli were delivered at 0 msec. C, A v-PrL neuron showed an antidromic response to BLA stimulation at a latency of $20 \mathrm{msec}$. In the first trace, five sweeps are superimposed. The lowest trace indicates a collision of an antidromic spike with a spontaneous spike $\left(^{*}\right)$.

those $(0.7 \pm 0.1 \mathrm{spikes} / \mathrm{sec} ; n=48)$ of neurons that revealed inhibitory or no responses $(t=2.20 ; p<0.05)$. Most (35 of 42 ; $83 \%$ ) neurons driven by CA1/S or BLA stimulation revealed no spontaneous activities. Spontaneously active neurons in the $\mathrm{V}-\mathrm{PrL}-\mathrm{MO}$ and IL revealed similar firing probabilities to $\mathrm{CA} 1 / \mathrm{S}(73 \pm 10 \% ; n=8)$ or BLA $(74 \pm 9 \% ; n=6)$ stimulation to those (CA1/S, $80 \pm 5 \%, n=22$; BLA, $77 \pm 5 \%, n=9$ ) of spontaneously nonactive neurons (CA1/S, $t=0.72, p=0.48$; BLA, $t=0.28, p=0.79$ ).

\section{Response to hippocampal stimulation}

Electrical stimulation of the CA1/S elicited both excitatory and inhibitory responses in the OMPFC (Table 1, Fig. 1). Most OMPFC neurons driven by CA1/S stimulation were located in the $\mathrm{v}$-PrL and IL, with only a few in the Cg1, d-PrL, MO, DP, or VO (Fig. 2 A). In contrast, OMPFC neurons that revealed inhibitory responses were located homogeneously in the OMPFC (Fig. 2A). The mean latency of excitatory responses of v-PrL-MO neurons (18.8 \pm 0.4 msec; $n=20$ ) was nearly the same as that of IL neurons $(21.0 \pm 1.2 \mathrm{msec} ; n=$ $19)$ and was significantly shorter than those of d-PrL $(30.5 \pm 6.7 \mathrm{msec} ; n=4$; $t=4.09$; $p<0.001)$. Cg1 neurons revealed much longer latencies $(48.0 \pm 4.9$ msec; $n=3$ ). The SDs of the excitatory latencies of OMPFC neurons driven by CA1/S stimulation were significantly correlated with excitatory latencies $(r=$ $0.87 ; p<0.0001 ; n=40)$, whereas there was no correlation between the firing probabilities and excitatory latencies $(r=-0.29 ; p=0.07 ; n=40)$ (Fig. 3A).

The mean latency of inhibitory responses of $\mathrm{v}$-PrL-MO neurons (22.5 \pm 5.6 msec; $n=8)$ to CA1/S stimulation was also shorter than that of Cg1 neurons (45.0 \pm 8.9 msec; $n=7 ; t=2.21 ; p<0.05)$. There was no regional difference in the duration of inhibitory responses of OMPFC neurons (mean, $196 \pm 26 \mathrm{msec} ; n=28$ ).

\section{Response to amygdalar stimulation}

OMPFC neurons were activated orthodromically (Fig. 1A-3) or antidromically (Fig. $1 C$ ) by BLA stimulation. The number of OMPFC neurons activated antidromically by BLA stimulation was increased by increasing stimulus currents of the BLA. The mean antidromic latency was $16.9 \pm$ $1.6 \mathrm{msec}(n=15)$. The mean threshold of BLA stimulation for the antidromic responses was $1.54 \pm 0.25 \mathrm{~mA}$ (range, $0.46-$ $3.0 \mathrm{~mA} ; n=13$ ). The mean threshold of BLA stimulation for the orthodromic responses was $0.28 \pm 0.02 \mathrm{~mA}$ (range, $0.10-$ $0.40 \mathrm{~mA} ; n=18$ ). Although 20 OMPFC neurons were orthodromically driven by BLA stimulation, 2 of the 20 neurons were activated by BLA stimulation at threshold currents (0.6 and $1.0 \mathrm{~mA})$ greater than the minimal current $(0.46 \mathrm{~mA})$ for antidromic activation. To exclude the possibility that the orthodromic responses of these two OMPFC neurons to BLA stimulation could be mediated by recurrent collaterals of OMPFC neurons, the data from these neurons were not included in later analyses. OMPFC neurons responding to BLA stimulation were primarily located in the $\mathrm{v}$-PrL and IL, as observed in the CA1/S stimulation experiments (Fig. $2 B$; Table 1). v-PrL-MO and IL neurons revealed short latencies $(16.0 \pm 1.9 \mathrm{msec}, n=6$ and $19.6 \pm 1.6 \mathrm{msec}, n=11$, respectively) of excitatory responses to BLA stimulation, which were similar to those of CA1/S stimulation. Latencies of Cg1 and d-PrL neurons were much longer $(\mathrm{Cg} 1,34.0 \pm 8.3 \mathrm{msec}, n=3$; d-PrL, $28.7 \pm 6.8$ msec, $n=3$ ). The SDs of the excitatory latencies of OMPFC 
A $\mathrm{CA} 1 / \mathrm{S}$ stimulation

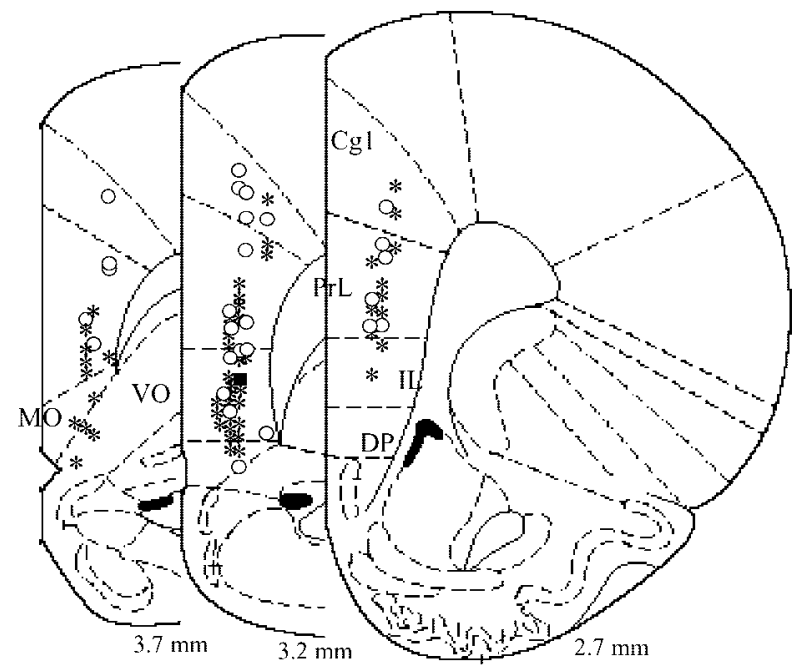

B BLA stimulation

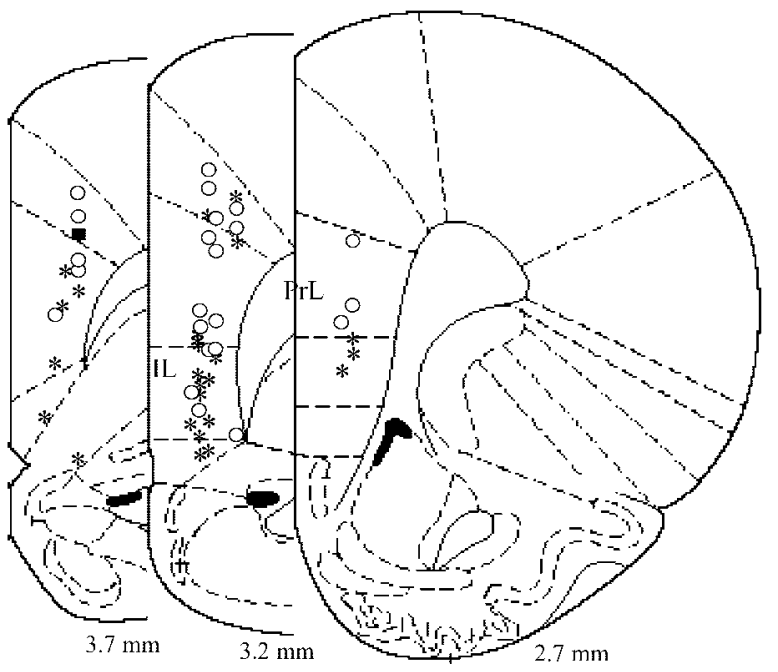

Figure 2. $A, B$, Distribution of OMPFC neurons responding to $C A 1 / S(A)$ and $B L A(B)$ stimulation. Neurons driven by $C A 1 / S$ stimulation $(*)$ were primarily located in the $\mathrm{v}-\mathrm{PrL}$ and IL, whereas neurons that revealed an inhibitory response to $C A 1 / S$ stimulation $(O)$ were located homogeneously in the OMPFC. A similar distribution was observed in neurons responding to BLA stimulation. The values represent the distance from bregma. *, Excitation; $\bigcirc$, inhibition;

口, excitation and inhibition.

neurons driven by BLA stimulation were significantly correlated with excitatory latencies $(r=0.85 ; p<0.0001 ; n=21)$, whereas no correlation could be found between the firing probabilities and excitatory latencies $(r=-0.14 ; p=0.53 ; n=21)$ (Fig. 3B).

The mean inhibitory latency of v-PrL-MO neurons $(20.8 \pm$ $2.7 \mathrm{msec} ; n=6)$ was significantly shorter than that of Cg1 (48.1 \pm 8.7 msec; $n=8 ; t=2.62 ; p<0.05)$. The mean duration of inhibitory responses to BLA stimulation $(184 \pm 19 \mathrm{msec} ; n=24)$ was similar to that of $\mathrm{CA} 1 / \mathrm{S}$ stimulation.

\section{Convergence and segregation of inputs within the OMPFC}

Both excitatory and inhibitory convergences of inputs from the CA1/S and BLA were examined. Of the 140 neurons studied, 18 (13\%) revealed excitatory responses to both CA1/S and BLA stimulation. In contrast, inhibitory convergence was found in 18 (64\%) of 28 neurons tested. Most of the neurons (78\%) driven by
A CAl/S stimulation
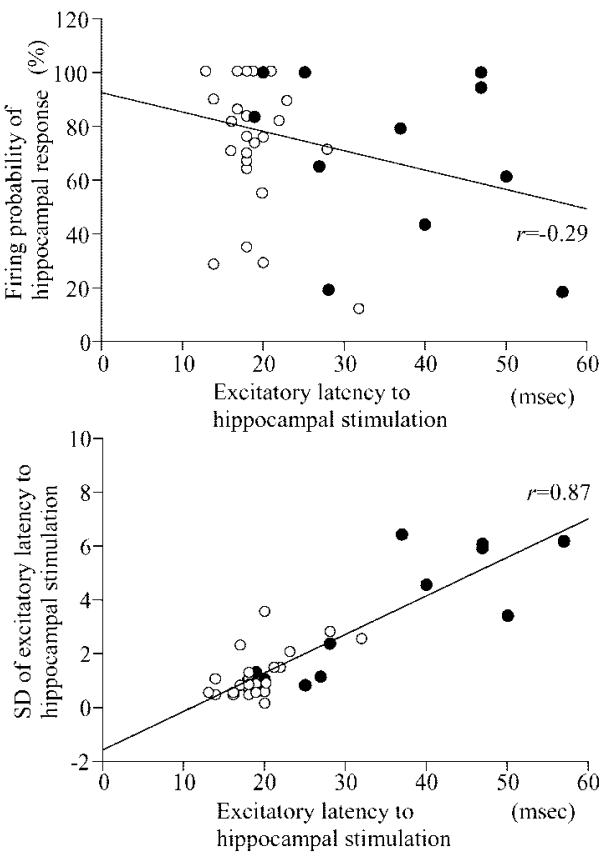

B BLA stimulation
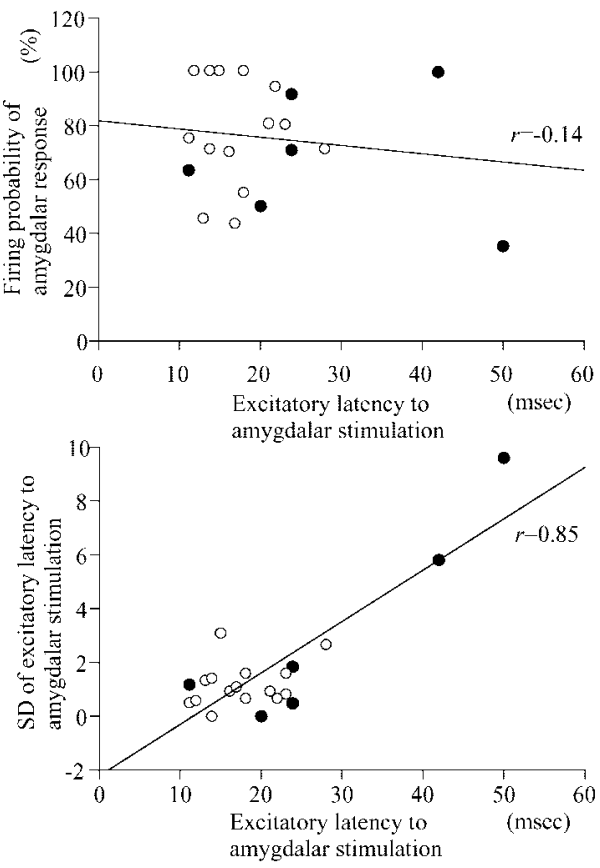

Figure 3. $A, B$, Relationship between excitatory latencies to $C A 1 / S(A)$ or $B L A(B)$ stimulation and firing probability (top) or SD of the excitatory latency (bottom). The excitatory latencies to $\mathrm{CA} 1 / \mathrm{S}$ or BLA stimulation were highly correlated with the SD of the latencies. $, \mathrm{Cg} 1, \mathrm{~d}-\mathrm{PrL}$, and DP-V0 neurons; $\bigcirc, \mathrm{v}-\mathrm{PrL}-\mathrm{M} 0$ and IL neurons. $\mathrm{v}-\mathrm{PrL}-\mathrm{M} 0$ and IL neurons are distributed in the range of small SDs.

both CA1/S and BLA stimulation were located in the v-PrL and IL (Fig. 4A). Thirty-two percent of IL neurons recorded revealed excitatory convergence of both inputs (Table 1). Neurons that revealed inhibitory response to both CA1/S and BLA stimulation were located homogeneously in the OMPFC. Additionally, the correlation between latencies to CA1/S and BLA stimulations was tested in OMPFC neurons showing either excitatory or inhibitory convergence of inputs from the CA1/S and BLA. The excitatory 


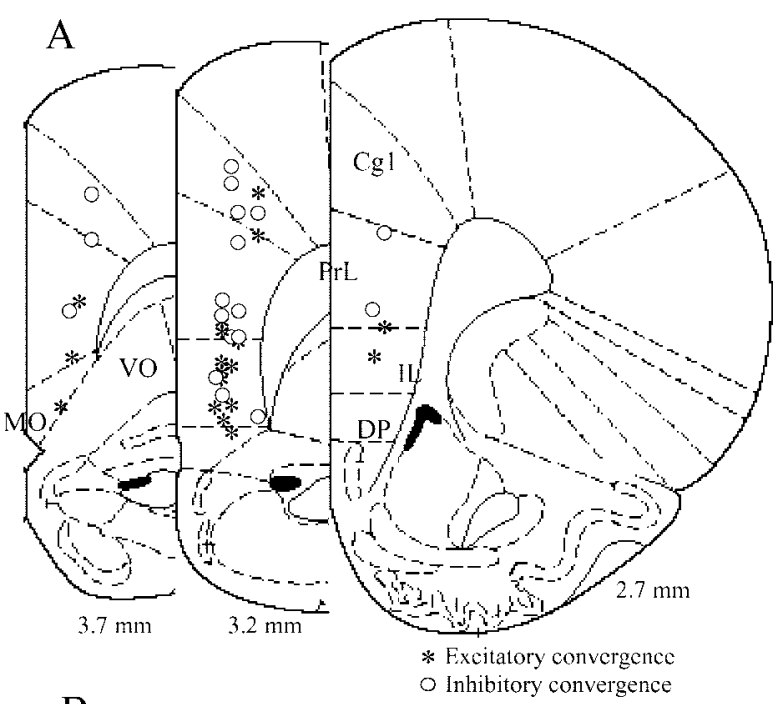

$\mathrm{B}$
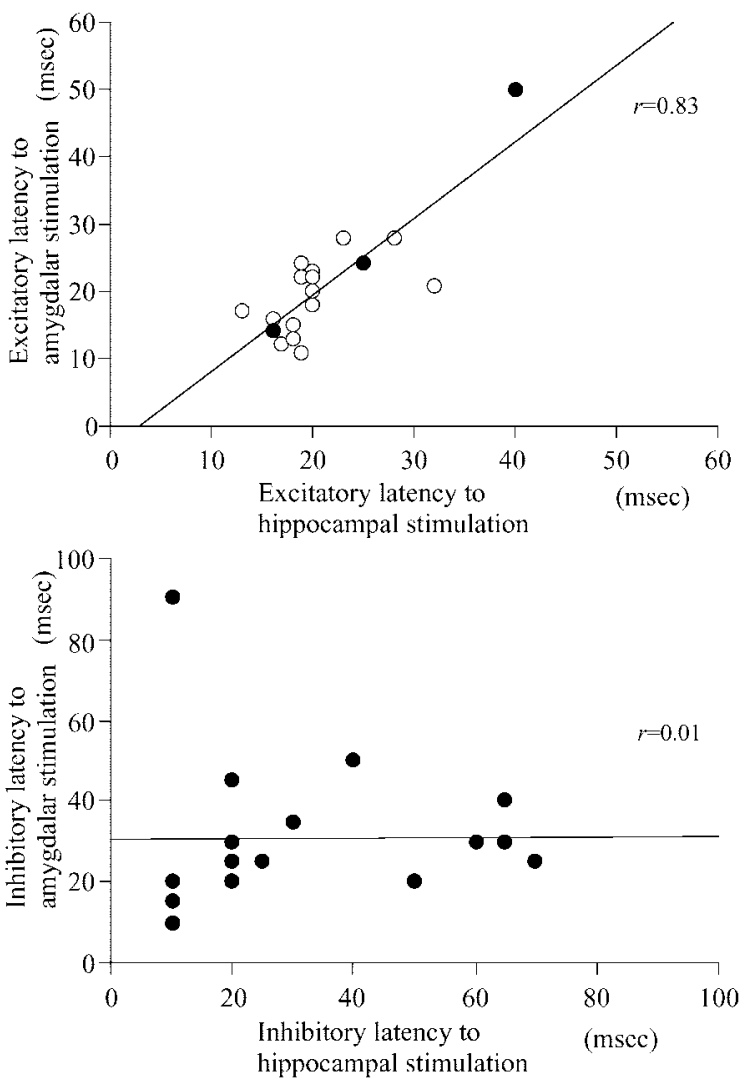

Figure 4. $\quad A, B$, Distribution of neurons showing excitatory and inhibitory convergences $(A)$ and the correlation of excitatory $(B$, top) or inhibitory ( $B$, bottom) latencies. Neurons driven by both CA1/S and BLA stimulation were primarily located in the v-PrL and IL. The excitatory but not inhibitory latencies to $C A 1 / S$ and BLA stimulation revealed a strong positive correlation. $A$, Asterisk, Neurons driven by both $C A 1 / S$ and BLA stimulation; $O$, neurons that revealed inhibitory responses to both stimulations. B, Top, O, v-PrL-M0 and ILneurons; $O, C g 1$, d-PrL, and DP neurons.

latencies to CA1/S stimulation were highly correlated with those of BLA stimulation ( $r=0.83 ; p<0.0001 ; n=18$ ) (Fig. $4 B$ ). In contrast, there was no significant correlation between the inhibitory latencies to CA1/S and BLA stimulation $(r=0.01 ; p=0.97$; $n=18$ ).
Lesions of reciprocal connections between the CA1/S and BLA To determine whether reciprocal connections between the CA1/S and BLA are involved in the responses of OMPFC neurons to CA1/S or BLA stimulation, the connections between the CA1/S and BLA were lesioned in 10 animals. In 4 of the 10 lesioned rats, in addition to CA1/S-evoked (or BLA-evoked) field potentials in the OMPFC, field potentials of the CA1/S (or BLA), evoked by BLA (or CA1/S) stimulation, were recorded. The remaining six rats were used for recording single-unit activity in the OMPFC. The apparently damaged regions included the ventral edge of the ventral hippocampus, amygdalohippocampal area (AHiPM), posteromedial cortical amygdaloid nucleus (PMCo), amygdalopiriform transition area (APir), and lateral entorhinal cortex (LEnt). CA1/S and BLA stimulation evoked characteristic negative-going field potentials in the OMPFC with a peak latency of 18-22 and 19-22 msec. Field potentials in the BLA responding to $\mathrm{CA} 1 / \mathrm{S}$ stimulation had two negative-going components, which revealed peak latencies of 5-15 and 24-40 msec. In the CA1/S, BLA stimulation evoked field potentials of monophasic negative waveform with a peak latency of 12-26 msec. Lesions of connections between the CA1/S and BLA produced marked decreases in the amplitudes of field potentials recorded in the CA1/S $\left(F_{(1,6)}=2.90 ; p<0.05 ; n=4\right)$ (Fig. $\left.5 C-1\right)$ and $\operatorname{BLA}\left(F_{(1,6)}=5.67\right.$; $p<0.01 ; n=4)$ (Fig. $5 B-1$ ) after stimulation of the BLA and CA1/S, respectively, whereas field potentials in the OMPFC evoked by CA1/S $\left(F_{(1,6)}=0.81 ; p=0.53 ; n=4\right)($ Fig. $5 B-2)$ and $\operatorname{BLA}\left(F_{(1,6)}=0.09 ; p=0.53 ; n=4\right)$ (Fig. $\left.5 C-2\right)$ stimulation revealed no apparent change in shape and amplitude. In six lesioned rats, in which the disconnection between the CA1/S and BLA was confirmed by a complete disappearance of field potentials of the CA1/S (or BLA) evoked by BLA (or CA1/S) stimulation, $28 \mathrm{OMPFC}$ neurons were recorded. Note that a majority $(n=19 ; 68 \%)$ were located in the IL. The excitatory latencies of these IL neurons to CA1/S ( $20.8 \pm 0.7 \mathrm{msec} ; n=12$; range, $15-24$ $\mathrm{msec})$ and BLA (19.9 $\pm 1.2 \mathrm{msec} ; n=13$; range, $12-26 \mathrm{msec})$ stimulation were similar to those in the nonlesioned rats (CA1/, $21.0 \pm 1.2 \mathrm{msec}, n=19$; BLA, $19.6 \pm 1.6 \mathrm{msec}, n=11$, respectively). Seven OMPFC neurons revealed excitatory convergence of CA1/S and BLA inputs and six of these neurons were located in the IL.

\section{Interaction of hippocampal and amygdalar inputs within the OMPFC}

Forty-four neurons ( $n=21$, nonlesioned rats; $n=23$, lesioned rats) in the OMPFC were tested for the interaction of CA1/S and BLA inputs. Conditioning stimulation of the BLA (or CA1/S) induced facilitatory $\left(\mathrm{BLA}-\mathrm{CA} 1 / \mathrm{S}: F_{(4,75)}=18.38, p<0.0001\right.$, $n=16$; CA1/S-BLA: $\left.F_{(4,45)}=14.49, p<0.0001, n=10\right)$ and inhibitory $\left(\mathrm{BLA}-\mathrm{CA} 1 / \mathrm{S}: F_{(6,112)}=13.55, p<0.0001, n=17\right.$; CA1/S-BLA: $F_{(6,84)}=12.28, p<0.0001, n=13$ ) effects on excitatory responses to CA1/S (or BLA) stimulation (Fig. 6). Because there was no difference in the effects of CA1/S (or BLA) conditioning stimulation on responses of OMPFC neurons to test stimulation of the BLA (or CA1/S) between the lesioned ( 6 animals, 23 neurons) and nonlesioned rats ( 5 animals, 21 neurons), the data from the two groups were pooled together. Twenty-one OMPFC neurons were tested for facilitatory effects of BLA (or CA1/S) conditioning stimulation on excitatory responses to CA1/S (or BLA) stimulation; 17 of these neurons revealed excitatory responses to both CA1/S and BLA stimulation, three were driven only by CA1/S stimulation, and one showed excitatory response only to BLA stimulation. When the test stimulus, which induced $\sim 10 \%$ firing probability in these OMPFC neurons, was 
given to the CA1/S (or BLA) after the conditioning subthreshold stimulus to the BLA (or CA1/S) with interstimulus intervals (ISIs) of $0,5,10$, and $20 \mathrm{msec}$, the firing probability was significantly higher than that after a single CA1/S (or BLA) stimulation (Fig. 6). In addition, inhibitory effects of BLA (or CA1/S) stimulation on the excitatory responses to CA1/S (or BLA) stimulation were examined in 30 OMPFC neurons. Six neurons showed excitatory convergence of the two inputs, 11 responded only to CA1/S stimulation, and 13 were driven only by BLA stimulation. Application of a conditioning BLA (or $\mathrm{CA} 1 / \mathrm{S}$ ) stimulus preceding a $\mathrm{CA} 1 / \mathrm{S}$ (or BLA) test stimulus with ISIs of 20 and 40 msec resulted in a marked decrease in the firing probability of the CA1/S-evoked (or BLA-evoked) responses as compared with a single CA1/S (or BLA) stimulus, which resulted in $\sim 90 \%$ firing probability (Fig. $6)$.

\section{Discussion}

In the present study, we show that OMPFC neurons receive excitatory and inhibitory convergence inputs from the hippocampus and amygdala. Excitatory convergence was observed primarily in the v-PrL and IL, whereas neurons showing inhibitory convergence were located homogeneously in the OMPFC. These observations indicate that both projections from the hippocampus and amygdala most likely cooperate in the v-PrL and IL. Furthermore, we demonstrated that excitatory responses of these neurons to hippocampal (or amygdalar) stimulation were greatly augmented by simultaneous stimulation of the amygdala (or hippocampus) and inhibited by amygdalar (or hippocampal) stimulation with a $20-40$ msec time lag. These results suggest that the timing of the two inputs is extremely important for the activity of $\mathrm{v}-\mathrm{PrL}$ and IL neurons. Because the facilitatory and inhibitory interactions between the amygdala (BLA) and hippocampus (CA1/S) were not altered after disconnection between the two brain areas, it is less likely that the modulatory effect of the two inputs occur via the BLA or CA1/S.

The projections of the ventral hippocampus and amygdala to the OMPFC have been investigated previously in anatomical (McDonald, 1987; Jay and Witter, 1991) and electrophysiological (Laroche et al., 1990; Pérez-Jaranay and Vives, 1991) studies; although, in these studies, the two pathways were studied independently. Regarding the hippocampal-OMPFC pathway, our results were consistent with previous studies (Laroche et al., 1990; Jay et al., 1992). v-PrL-MO neurons revealed the shortest excitatory latency, whereas the latencies were longer in the $\mathrm{Cg} 1$ and $\mathrm{d}-\mathrm{PrL}$. Because previous studies reported that the innervation of hip-

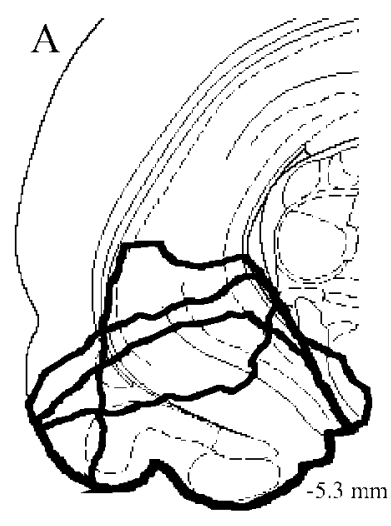

B-1
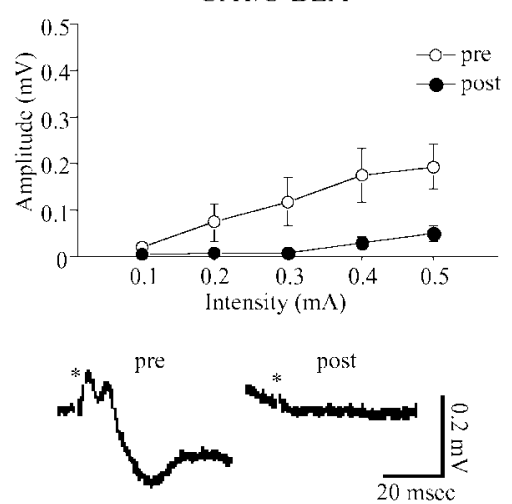

C-1
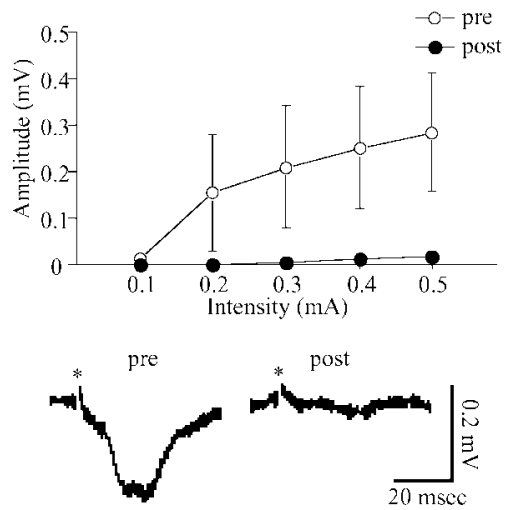
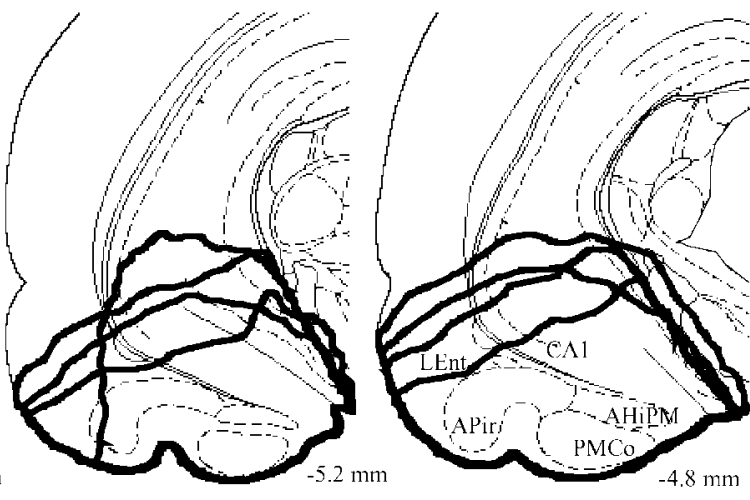

B-2
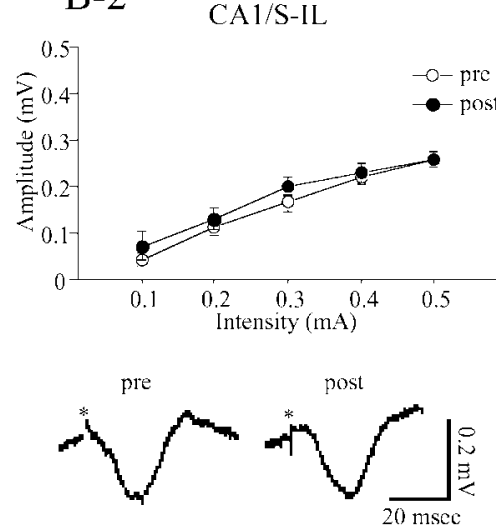

$\mathrm{C}-2$

BLA-IL
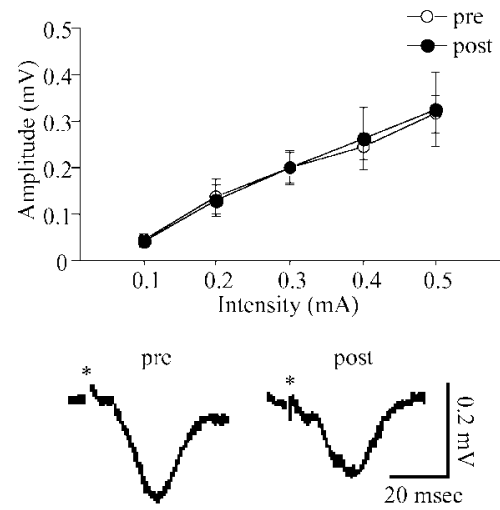

Figure 5. Extent of lesioned sites $(A)$ and effects of the lesions on field potentials of the BLA, CA1/S, and IL evoked by CA1/S ( $B)$ or BLA ( $C$ ) stimulation. $A$, Lesioned areas characterized by apparent loss of tissue integrity and bleeding were enclosed by thick lines (4 rats), all of which were along the base of the brain and included the ventral edge of the ventral hippocampus, AHiPM, PMCo, APir, and LEnt. The values represent the distance from bregma. B, C, Top, The relationship between stimulus intensity and amplitudes of field potentials. Open and filled circles show amplitudes of the field potentials before and after the lesions, respectively. Bottom, Samples of field potentials evoked by each stimulation at $0.3 \mathrm{~mA}$ with a $0.1 \mathrm{msec}$ duration before (left) and after (right) the lesions. Asterisks show the time of stimulation. Regarding the field potentials of the BLA evoked by CA1/S stimulation $(B-1)$, the amplitudes of the second negative-going component are plotted (top).

pocampal efferents was less dense in the d-PrL and Cg1 than in the v-PrL-MO (Jay and Witter, 1991), and that there are reciprocal intrinsic projections among the subregions of the OMPFC (Condé et al., 1995; Fisk and Wyss, 1999), the long excitatory latencies of Cg1 and d-PrL neurons are thought to be attributable to polysynaptic intracortical conduction. The larger SDs of the long excitatory latencies to CA1/S stimulation support polysynaptic activation of these neurons. Previous intracellular recordings of PrL neurons indicated that CA1/S stimulation resulted in 

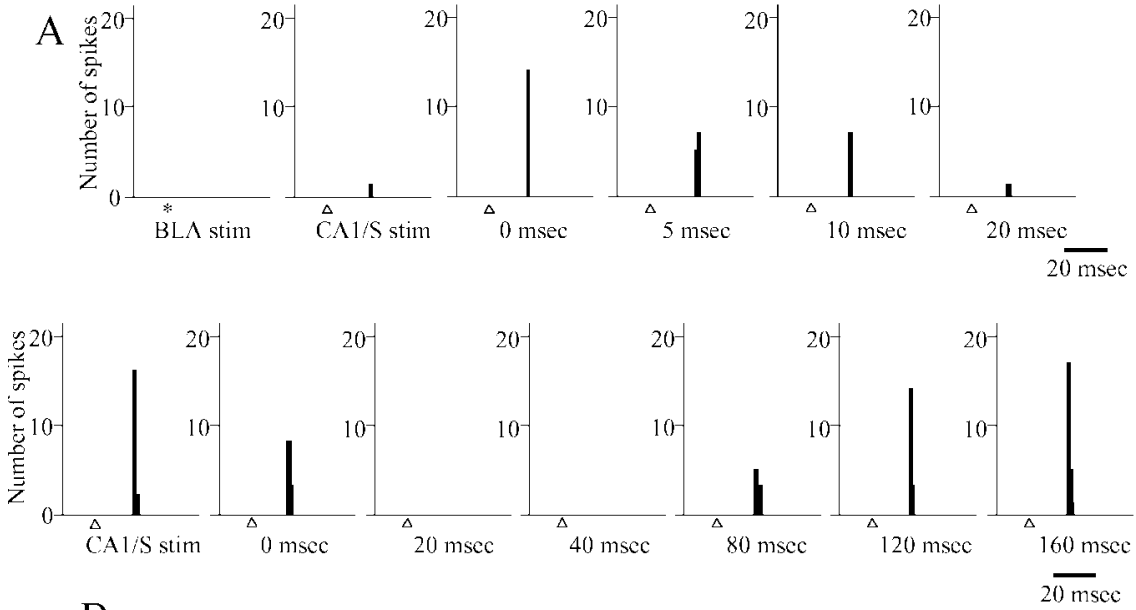

B
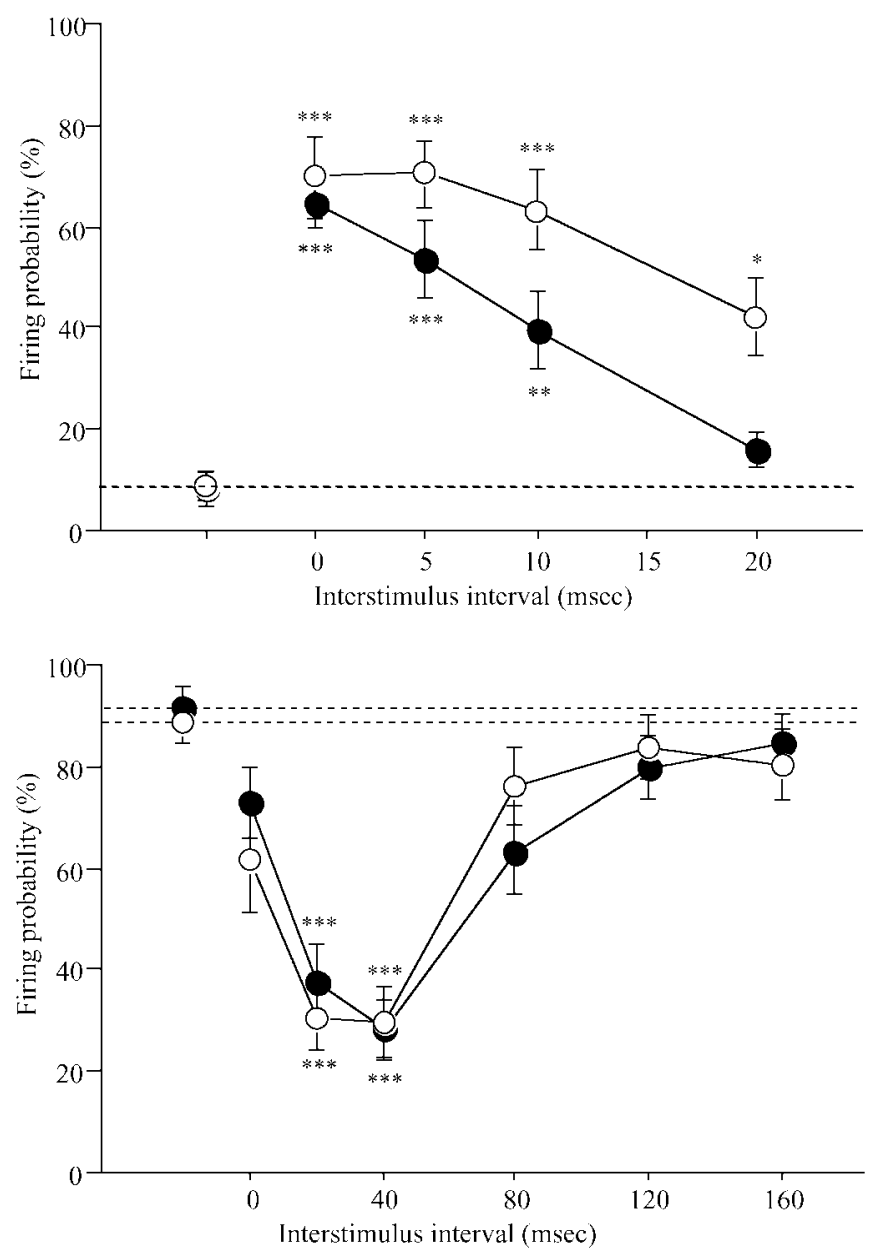

Figure 6. Examples of OMPFC neurons that revealed facilitation $(A$, top $)$ and inhibition $(A$, bottom) of the firing probability of CA1/S-induced excitatory responses after the conditioning stimulation of the BLA and the time course of the facilitatory $(B$, top $)$ and inhibitory ( $B$, bottom) effects of $B L A$ (or $C A 1 / S$ ) stimulation on excitatory responses to $C A 1 / S$ (or BLA) stimulation. $A$, These recordings were obtained in the lesioned rats in which field potentials of the $C A 1 / S$ (or BLA) evoked by BLA (or CA1/S) stimulation completely disappeared after the lesions. These neurons were located in the IL $(A, \operatorname{top})$ and v- $\operatorname{PrL}\left(A\right.$, bottom), respectively. ${ }^{*}$, BLA stimulation; $\Delta$, CA1/S stimulation. The time under PSTHs shows ISIs. The PSTH in the left end at the top represents responses to subthreshold BLA stimulation, which by itself evoked no response. The second PSTH from the left end at the top and PSTH in the left end at the bottom show responses to single CA1/S stimulation. $B$, In each graph, filled (top, $n=16$; bottom, $n=17$ ) and open (top, $n=10$; bottom, $n=13$ ) circles represent firing probability of OMPFC neurons to CA1/S and BLA stimulation, respectively. The filled and open circles of the left end and horizontal dotted lines represent firing probability to a single CA1/S and BLA stimulation. ${ }^{*} p<0.05 ;{ }^{* *} p<0.01 ;{ }^{* *} p<0.0001$; compared with firing probability to single CA1/S or BLA stimulation. an early EPSP followed by a prolonged phase of hyperpolarization (Thierry et al., 2000). The long inhibitory responses observed in this study are likely to be attributable to this hyperpolarization, which may be induced by the activation of adjacent GABAergic interneurons via monosynaptical glutamatergic inputs from the CA1/S.

These experiments demonstrated a distinctive localization of OMPFC neurons, which revealed excitatory responses to BLA stimulation, although a previous study did not demonstrate this apparent distribution of the excitatory responses (Pérez-Jaranay and Vives, 1991). In this study, the neurons driven by BLA stimulation were encountered predominantly in the v-PrL and IL. These findings are consistent with anatomical findings that the PrL and IL receive major inputs from the BLA (McDonald, 1987; Condé et al., 1995), particularly from the posterior part, and that BLA neurons projecting to the OMPFC contain excitatory neurotransmitters (McDonald, 1996). Our success in demonstrating the topographical distribution of BLA-driven neurons may be explained by the fact that the stimulating sites of the BLA in our study were localized in a more posterior part of the BLA than in the studies by Pérez-Jaranay and Vives (1991). Excitatory latencies of OMPFC neurons to BLA stimulation in this study $(>11 \mathrm{msec})$ were longer than those reported by Pérez-Jaranay and Vives $(<10$ $\mathrm{msec}$ ). Because antidromic latencies of BLA neurons to stimulation of the MPFC were $\sim 10-26$ msec (Rosenkranz and Grace, 2001), orthodromic excitatory latencies of MPFC neurons to BLA stimulation is expected to be longer than $10 \mathrm{msec}$. Inhibitory responses to BLA stimulation were more commonly and diffusely observed within the entire OMPFC, as reported previously (Pérez-Jaranay and Vives, 1991). Given that BLA neurons projecting to the OMPFC are glutamatergic (McDonald, 1996), indirect pathways are thought to mediate these inhibitory responses. It is most likely that activation of IL (or v-PrL) neurons by excitatory BLA inputs triggers an intracortical inhibitory circuit within the OMPFC.

In accordance with previous findings of reciprocal connections between the OMPFC and BLA (Sesack et al., 1989; Pérez-Jaranay and Vives, 1991; McDonald et al., 1996; Rosenkranz and Grace, 2001), OMPFC neurons were activated orthodromically or antidromically by BLA stimulation. The thresholds for the orthodromic responses of OMPFC neurons to 
BLA stimulation (mean, $0.28 \mathrm{~mA}$ ) were much lower than those for the antidromic responses (mean, $1.54 \mathrm{~mA}$ ). This suggests that BLA-induced orthodromic responses of OMPFC neurons were attributable to direct activation of BLA neurons projecting to the OMPFC, but not attributable to activation of the recurrent axon collaterals of OMPFC neurons.

Anatomical (Krettek and Price, 1977a; Canteras and Swanson, 1992; Pitkanen et al., 2000) and physiological (Colino and Fernández de Molina, 1986; Maren and Fanselow, 1995) evidence have demonstrated that there are reciprocal connections between the CA1/S and BLA. Thus, it is possible that CA1/S (or BLA) stimulation induced the excitatory responses of OMPFC neurons either via the CA1/S-BLA (or BLA-CA1/S)-OMPFC pathway or by activating axon collaterals of BLA (or CA1/S) neurons projecting simultaneously to the CA1/S (or BLA) and OMPFC. To exclude this possibility, the reciprocal circuits between the CA1/S and BLA were disconnected by discrete lesions of the areas including the ventral edge of the ventral hippocampus, AHiPM, PMCo, APir, and LEnt, in which the reciprocal fibers from the CA1/S and BLA are reported to pass through (Krettek and Price, 1977a; Canteras and Swanson, 1992; Pitkanen et al., 2000). Because field potentials recorded in the BLA (or CA1/S) completely disappeared after lesions of the reciprocal connections, it is less likely that the excitatory responses of OMPFC neurons to CA1/S (or BLA) stimulation were induced via the BLA (or CA1/S). Furthermore, observations of excitatory convergence and facilitatory and inhibitory interactions of the two inputs from the CA1/S and $\mathrm{BLA}$ in the lesioned rats indicate that the convergence and interaction did not occur via the CA1/S and BLA.

Previous studies have demonstrated that activation of the OMPFC, particularly the IL, is involved in fear reduction and extinction of learned fear (Garcia et al., 1999; Herry and Garcia, 2002; Milad and Quirk, 2002). This is thought to occur because of the suppression of the activity of BLA neurons, which is caused by activation of inhibitory interneurons of the BLA by excitatory OMPFC inputs (Rosenkranz and Grace, 2001). In contrast, when neuronal activity in the OMPFC is inhibited by BLA inputs, fear responses can be enhanced because of the disinhibition of the OMPFC-BLA circuit responsible for fear reduction and extinction of learned fear (Garcia et al., 1999). In this study, we found that many OMPFC neurons, which were inhibited by BLA stimulation, also revealed an apparent inhibition by CA1/S stimulation. Although this finding suggests involvement of the hippocampus in the fear-enhancing circuit, the physiological significance of the inhibitory influence of the hippocampus on OMPFC neurons remains to be determined.

In the present experiments, excitatory convergence of the two inputs from the CA1/S and BLA was observed predominantly in the $\mathrm{V}$-PrL and IL. This is consistent with the results of a retrograde tract-tracing study, which revealed that the number of retrogradely labeled cells in the CA1/S and BLA was greater when the injection sites were located in the ventral portion of the MPFC, including the IL (Condé et al., 1995). Behavioral and physiological studies have demonstrated that the PrL and IL are both involved in learning and memory performance (Kesner et al., 1996; Ragozzino et al., 1998; Baldwin et al., 2002). The v-PrL and IL have been considered to play major roles in regulating autonomic activity, because these regions are densely connected with several subcortical areas involved in autonomic functions (Terreberry and Neafsey, 1987; Takagishi and Chiba, 1991; Owens and Verberne, 1996; Ishikawa et al., 2001). In addition, amygdala is also the major target of neurons originating in v-PrL and IL (Takagishi and Chiba, 1991; McDonald et al., 1996). Therefore, the excitatory convergence of v-PrL and IL neurons from hippocampus and amygdala may play roles in learning and memory performance, which are closely associated with emotional and autonomic responses.

The excitatory latencies of IL neurons to CA1/S and BLA stimulation were approximately the same ( $\sim 20 \mathrm{msec})$, and a marked augmentation of excitatory responses of IL neurons occurred when stimulation of the two brain sites was given simultaneously. These findings suggest the importance of the timing of excitatory inputs to the IL from the hippocampus and amygdala. If activation of amygdalar neurons projecting to a given IL neuron occurs simultaneously with excitation of hippocampal neurons projecting to the same IL neuron, the hippocampal input to the IL neuron could potentially be significantly amplified. This may be associated with better memory consolidation of matters or events accompanying emotional responses. Furthermore, considering that synchronized firings of neurons in different brain regions are crucial for cognitive brain mechanisms (Engel et al., 1992, 1999; Singer, 1999), amplification of neuronal activities in the OMPFC by synchronized firing of hippocampal and amygdalar neurons may be significant for learning and memory performance.

\section{References}

Bacon SJ, Headlam AJ, Gabbott PL, Smith AD (1996) Amygdala input to medial prefrontal cortex (mPFC) in the rat: a light and electron microscope study. Brain Res 720:211-219.

Baldwin AE, Sadeghian K, Kelley AE (2002) Appetitive instrumental learning requires coincident activation of NMDA and dopamine D1 receptors within the medial prefrontal cortex. J Neurosci 22:1063-1071.

Canteras NS, Swanson LW (1992) Projections of ventral subiculum to the amygdala, septum and hypothalamus: a PHAL anterograde tract-tracing study in the rat. J Comp Neurol 324:180-194.

Colino A, Fernández de Molina A (1986) Electrical activity generated in subicular and entorhinal cortices after electrical stimulation of the lateral and basolateral amygdala of the rat. Neuroscience 19:573-580.

Condé F, Maire-Lepoivre E, Audinat E, Crépel F (1995) Afferent connections of the medial frontal cortex of the rat. II. Cortical and subcortical afferents. J Comp Neurol 352:567-593.

Engel AK, Fries P, Konig P, Brecht M, Singer W (1999) Temporal binding, binocular rivalry, and consciousness. Conscious Cogn 8:128-151.

Engel AK, Konig P, Kreiter AK, Schillen TB, Singer W (1992) Temporal coding in the visual cortex: new vistas on integration in the nervous system. Trends Neurosci 15:218-226.

Fisk GD, Wyss JM (1999) Associational projections of the anterior midline cortex in the rat: intracingulate and retrosplenial connections. Brain Res $825: 1-13$.

Floresco SB, Seamans JK, Phillips AG (1997) Selective roles for hippocampal, prefrontal cortical, and ventral striatal circuits in radial-arm maze tasks with or without a delay. J Neurosci 17:1880-1890.

Gaffan D, Murray EA, Fabre-Thorpe M (1993) Interaction of the amygdala with the frontal lobe in reward memory. Eur J Neurosci 5:968-975.

Garcia R, Vouimba RM, Baudry M, Thompson RF (1999) The amygdala modulates prefrontal cortex activity relative to conditioned fear. Nature 402:294-296.

Herry C, Garcia R (2002) Prefrontal cortex long-term potentiation, but not long-term depression, is associated with the maintenance of extinction of learned fear in mice. J Neurosci 22:577-583.

Ishikawa A, Nakamura S (2002) Convergence and interaction of hippocampal and amygdalar inputs to the medial prefrontal cortex in rats. Neurosci Res [Suppl] 26:S130.

Ishikawa A, Ishida Y, Nakamura S (2001) Neurons in the lateral paragigantocellular nucleus projecting to the infralimbic cortex in rats: electrical activity and response to changes in blood pressure. Neurosci Lett 311:57-60.

Jay TM, Witter MP (1991) Distribution of hippocampal CA1 and subicular efferents in the prefrontal cortex of the rat studied by means of anterograde transport of Phaseolus vulgaris-leucoagglutinin. J Comp Neurol 313:574-586.

Jay TM, Thierry AM, Wiklund L, Glowinski J (1992) Excitatory amino acid 
pathway from the hippocampus to the prefrontal cortex. Contribution of AMPA receptors in hippocampo-prefrontal cortex transmission. Eur J Neurosci 4:1285-1295.

Jinks AL, McGregor IS (1997) Modulation of anxiety-related behaviours following lesions of the prelimbic or infralimbic cortex in the rat. Brain Res 772:181-190.

Kesner RP, Hunt ME, Williams JM, Long JM (1996) Prefrontal cortex and working memory for spatial response, spatial location, and visual object information in the rat. Cereb Cortex 6:311-318.

Krettek JE, Price JL (1977a) Projections from the amygdaloid complex and adjacent olfactory structures to the entorhinal cortex and to the subiculum in the rat and cat. J Comp Neurol 172:723-752.

Krettek JE, Price JL (1977b) Projections from the amygdaloid complex to the cerebral cortex and thalamus in the rat and cat. J Comp Neurol 172:687-722.

Laroche S, Jay TM, Thierry AM (1990) Long-term potentiation in the prefrontal cortex following stimulation of the hippocampal CA1/subicular region. Neurosci Lett 114:184-190.

Maren S, Fanselow MS (1995) Synaptic plasticity in the basolateral amygdala induced by hippocampal formation stimulation in vivo. J Neurosci 15:7548-7564.

McDonald AJ (1996) Glutamate and aspartate immunoreactive neuron of the rat basolateral amygdala: colocalization of excitatory amino acids and projections to the limbic circuit. J Comp Neurol 365:367-379.

McDonald AJ (1987) Organization of amygdaloid projections to the mediodorsal thalamus and prefrontal cortex: a fluorescence retrograde transport study in the rat. J Comp Neurol 262:46-58.

McDonald AJ, Mascagni F, Guo L (1996) Projections of the medial and lateral prefrontal cortices to the amygdala: a Phaseolus vulgaris leucoagglutinin study in the rat. Neuroscience 71:55-75.

Milad MR, Quirk GJ (2002) Neurons in medial prefrontal cortex signal memory for fear extinction. Nature 420:70-74.

Morgan MA, Romanski LM, LeDoux JE (1993) Extinction of emotional learning: contribution of medial prefrontal cortex. Neurosci Lett 163:109-113.

Owens NC, Verberne AJM (1996) An electrophysiological study of the me- dial prefrontal cortical projection to the nucleus of the solitary tract in rat. Exp Brain Res 110:55-61.

Paxinos G, Watson C (1998) The rat brain in stereotaxic coordinates, Ed 4. San Diego: Academic.

Pérez-Jaranay JM, Vives F (1991) Electrophysiological study of the response of medial prefrontal cortex neurons to stimulation of the basolateral nucleus of the amygdala in the rat. Brain Res 564:97-101.

Pitkanen A, Pikkarainen M, Nurminen N, Ylinen A (2000) Reciprocal connections between the amygdala and the hippocampal formation, perirhinal cortex, and postrhinal cortex in rat. A review. Ann NY Acad Sci 911:369-391.

Quirk GJ, Russo GK, Barron JL, Lebron K (2000) The role of ventromedial prefrontal cortex in the recovery of extinguished fear. J Neurosci 20:6225-6231.

Ragozzino ME, Adams S, Kesner RP (1998) Differential involvement of the dorsal anterior cingulate and prelimbic-infralimbic areas of the rodent prefrontal cortex in spatial working memory. Behav Neurosci 112:293-303.

Rosenkranz JA, Grace AA (2001) Dopamine attenuates prefrontal cortical suppression of sensory inputs to the basolateral amygdala of rats. J Neurosci 21:4090-4103.

Sesack SR, Deutch AY, Roth RH, Bunney BS (1989) Topographical organization of the efferent projections of the medial prefrontal cortex in the rat: an anterograde tract-tracing study with Phaseolus vulgaris leucoagglutinin. J Comp Neurol 290:213-242.

Singer W (1999) Neuronal synchrony: a versatile code for the definition of relations? Neuron 24:111-125.

Takagishi M, Chiba T (1991) Efferent projections of the infralimbic (area $25)$ region of the medial prefrontal cortex in the rat: anterograde tracer PHA-L study. Brain Res 566:26-39.

Terreberry RR, Neafsey EJ (1987) The rat medial frontal cortex projects directly to autonomic regions of the brainstem. Brain Res Bull 19:639-649.

Thierry AM, Gioanni Y, Dégénétais E, Glowinski J (2000) Hippocampoprefrontal cortex pathway: anatomical and electrophysiological characteristics. Hippocampus 10:411-419. 